\title{
Magnetic Characterisation of Ni Triangular Elements Prepared by Nanosphere Lithography
}

\author{
H. GŁACZYŃSKA ${ }^{a, b}, \mathrm{U} . \operatorname{EBELS}^{a}$, J. RYBCZYŃSKI ${ }^{b}$, \\ W. KandULSKI ${ }^{b}$ AND M. GIERSIG ${ }^{b}$ \\ ${ }^{a}$ SPINTEC (URA 2512 CNRS/CEA), CEA/Grenoble \\ 17 Av. Martyrs, 38054 Grenoble Cedex 9, France \\ ${ }^{b}$ Center of Advanced European Studies And Research, \\ CAESAR/Ludwig-Erhard-Allee 2, 53175 Bonn, Germany
}

\begin{abstract}
A systematic study of the magnetic properties of submicron equilateral $\mathrm{Ni}$ triangles is reported. The triangular shape is a result of the particular preparation method used, which is based on a new kind of nanosphere lithography technique. In this case, the magnetic material is deposited through a latex sphere mask, yielding a periodic hexagonal array of in-plane magnetised triangular shaped elements. The magnetic properties were investigated as a function of thickness by magnetic force microscopy, superconducting quantum interference device magnetometry and vibrating sample magnetometry.
\end{abstract}

PACS numbers: 75.75.+a, 75.60.Ej, 61.46.+w

\section{Introduction}

Continual increase in the areal density of data storage elements [1, 2] has initiated the exploration of alternative lithography methods to fabricate sub-micron and nanometer sized elements, arranged in ordered arrays.

Here we make use of a new kind lithography technique [3] which is based on the deposition and ordering of polystyrene (PS) particles on a water surface. Similarly to Langmuir-Blodgett films, the polystyrene particle array is then transferred to a substrate surface. This mask preparation method is distinguished by a short preparation time, a high level of hexagonal order of the PS particles and the large size of defect free areas. Furthermore, the variation of the PS diameter allows one to create structures over a $\mathrm{cm}^{2}$ area with controlled size, shape, and 


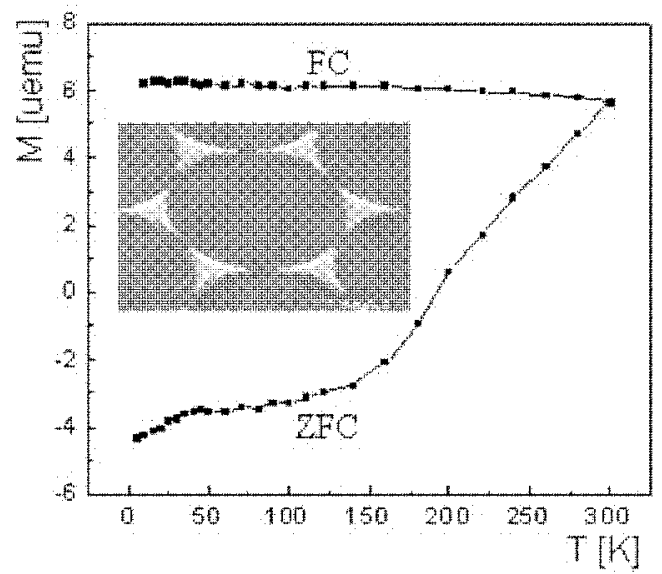

Fig. 1. The ZFC and FC temperature dependence (at $H=20 \mathrm{Oe}$ ) of the magnetisation for $10 \mathrm{~nm}$ thick Ni elements, evaporated through a $1710 \mathrm{~nm}$ PS mask. The inset shows an SEM image taken at a non-perpendicular angle to plane of the triangular elements.

also of varying interparticle spacing. Subsequent deposition of the magnetic material by means of electron beam evaporation and lift-off of the PS spheres results in a honeycomb pattern of polycrystalline magnetic elements, which precisely fill the space between the latex spheres, see the scanning electron microscopy (SEM) image (taken at non-perpendicular incidence) in the inset of Fig. 1.

The deposition through this kind of mask differs from conventional techniques, where the lateral size of the final element is commonly larger than the thickness of the mask through which the material is deposited. In contrast, here the lateral element size is approximately half the mask thickness which is given by the PS diameter. This might affect the quality of the deposition and thus of the magnetic properties of the final elements. Therefore a systematic characterisation of the magnetic properties is essential for the optimisation of such a technique. Besides this, the arrays of triangular shaped elements provide an interesting model system for the study of the micromagnetic configurations of a submicron system. These configurations depend sensitively on the element lateral size, element thickness, as well as on the exact shape. So far only few investigations have been reported for equilateral triangular elements [4, 5].

The following study has been carried out for Ni triangles of $10,20,30$, and $40 \mathrm{~nm}$ thickness evaporated through a PS mask of $1710 \mathrm{~nm}$ diameter. This yields a lateral element size of about $820 \mathrm{~nm}$ with an element spacing of $200 \mathrm{~nm}$. Results are presented on the temperature dependence of the magnetisation obtained by SQUID magnetometry, on the hysteresis loops studied by vibrating sample magnetometry (VSM) as well as results from magnetic force microscopy (MFM) imaging which are compared to micromagnetic simulations. 


\section{Temperature dependence of the magnetisation}

Temperature dependent magnetisation measurements have been performed using SQUID magnetometry to check for ferromagnetic/superparamagnetic behaviour. In Fig. 1 the zero field cooled (ZFC) and field cooled (FC) magnetisation curves are shown representatively for $t=10 \mathrm{~nm}$, measured in a field of $20 \mathrm{Oe}$ applied in the element plane. The FC branch is relatively constant in this temperature range, consistent with ferromagnetic behaviour. Similarly, the ZFC branch varies little or is constant to about $150 \mathrm{~K}$ and then rises up to the $\mathrm{FC}$ branch. The increase is interpreted as a realignment of the magnetic moments, when the external field becomes smaller than the temperature dependent coercive field $H_{\mathrm{c}}(T)$. The broad transition range from 150 to $300 \mathrm{~K}$ corresponds then to a relatively large distribution of the saturation field/coercive field. The small variation in the ZFC branch below $50 \mathrm{~K}$ is not yet understood. It might be attributed to the presence of a small volume fraction of either a ferromagnetic or a superparamagnetic phase.

\section{Room temperature magnetisation as a function of element thickness}

The broad transition range observed in the temperature dependent data is consistent with room temperature (in-plane) VSM hysteresis measurements shown in Fig. 2 for different thicknesses. These curves consist of two parts: a high field rotational part and a low field irreversible switching part. The irreversible switching field range, corresponding to the reversal of the ferromagnetic parts, is relatively large, extending to over 100 Oe. The rotational part might have different origins which remain to be elucidated: (i) it either corresponds to a superparamagnetic phase or (ii) to ferromagnetic regions that are hard to saturate. In both cases we suspect that it originates in the triangular corners which are very narrow, and for which the deposition will lead to strong disorder as well as a reduced thickness. Hence the ferromagnetic order might be influenced and lead to superparamagnetic behaviour. On the other hand, if ferromagnetic order is established, these triangular corners will correspond to regions that are hard to saturate, due to the strong demagnetisation effects, when the field is applied perpendicular to the edge. One might therefore describe the triangular elements by a ferromagnetic core of approximately circular shape which is to a certain extent decoupled from ferromagnetic/superparamagnetic corners that respond differently to an applied field. Within this picture, the absence of an in-plane angular dependence of the loop shape, the coercive field, and the remanence might be explained. It is noted that for ideal triangular elements a configurational anisotropy of sixfold symmetry has been confirmed [5] whereby the inhomogeneous demagnetisation fields induce slightly different magnetisation distributions for different magnetisation directions. 


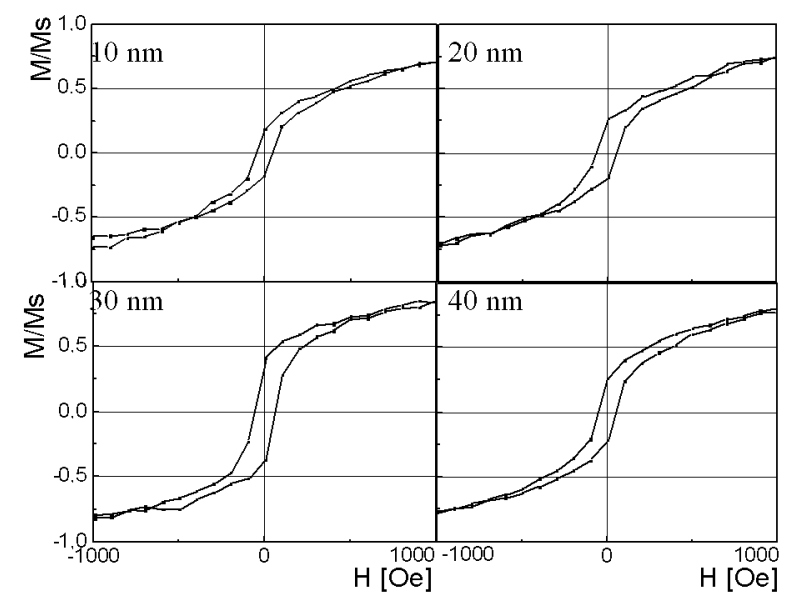

Fig. 2. Room temperature VSM magnetisation curves for Ni elements as a function of thickness: $10 \mathrm{~nm}, 20 \mathrm{~nm}, 30 \mathrm{~nm}, 40 \mathrm{~nm}$.

The thickness dependence of the normalized remanent magnetisation $M_{\mathrm{r}}$, the coercive field $H_{\mathrm{c}}$ and the in-plane saturation field $H_{\text {sat }}$ for the $M-H$ loops of Fig. 2, are given in Table. It is noted that from the VSM studies it is found that the in-plane saturation is always lower than the out of plane saturation, confirming that the magnetisation is well aligned in the sample plane as expected for the low aspect ratio of thickness to lateral size $(0.012-0.05)$.

\section{TABLE}

Comparison of $M_{\mathrm{r}}, H_{\mathrm{c}}$, and $H_{\mathrm{sat}}$ for different thickness of the nickel elements.

\begin{tabular}{c|c|c|c}
\hline \hline Element thickness [nm] & $M_{\mathrm{r}} / M_{\mathrm{s}}$ & $H_{\mathrm{c}}[\mathrm{Oe}]$ & $H_{\text {sat }}[\mathrm{Oe}]$ \\
\hline 10 & 0.18 & 50 & 3783 \\
20 & 0.23 & 59 & 3261 \\
30 & 0.40 & 60 & 3000 \\
40 & 0.22 & 50 & 3600
\end{tabular}

From the thickness dependence it is noted that all parameters $M_{\mathrm{r}}, H_{\mathrm{c}}$, and $H_{\text {sat }}$ reveal a similar behaviour. The remanence and coercive field first increase up to $30 \mathrm{~nm}$ and then decrease, while the saturation field first decreases and then increases again. This oscillating behaviour is somewhat unexpected from a micromagnetics point of view, but correlates with the magnetic contrast found by MFM imaging after saturation in an in-plane field of $H=3 \mathrm{kOe}$. The corresponding MFM images in Fig. 3 (top row), taken at zero field, reveal different types of contrasts that are interpreted as either a flux closure state $(10 \mathrm{~nm}, 40 \mathrm{~nm})$ or as a disordered single domain state $(30 \mathrm{~nm})$, where the orientation of the single domain contrast varies from one element to the other. For the $10 \mathrm{~nm}, 20 \mathrm{~nm}$, and $30 \mathrm{~nm}$ 

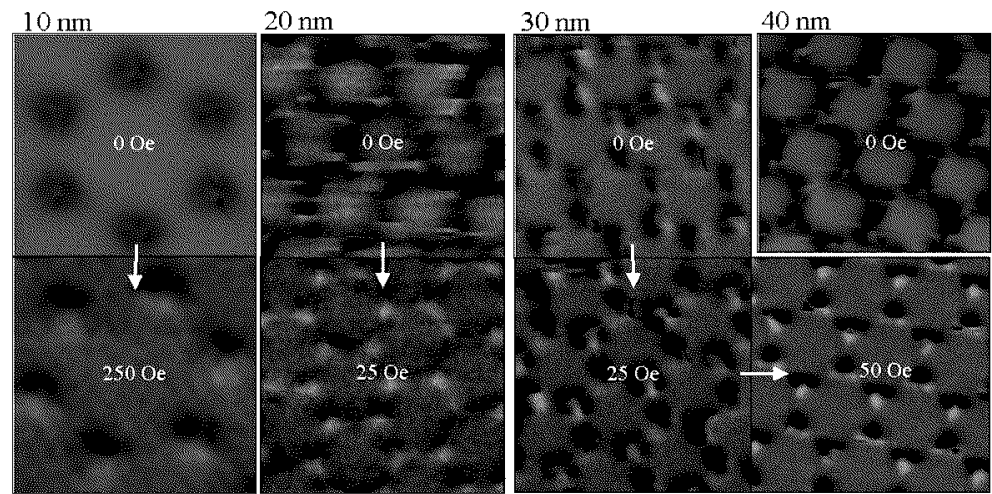

Fig. 3. Zero field MFM images as a function of thickness (top row) and field dependence of the MFM contrast (bottom row) for the $10 \mathrm{~nm}\left(3 \times 3 \mu \mathrm{m}^{2}\right)$, the $20 \mathrm{~nm}\left(6 \times 6 \mu \mathrm{m}^{2}\right)$, the $30 \mathrm{~nm}\left(6 \times 6 \mu \mathrm{m}^{2}\right)$ and the $40 \mathrm{~nm}\left(6 \times 6 \mu \mathrm{m}^{2}\right)$ Ni triangles.

a)

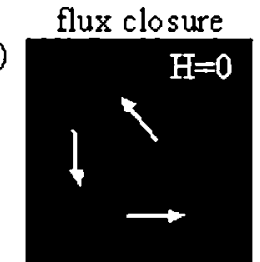

b)

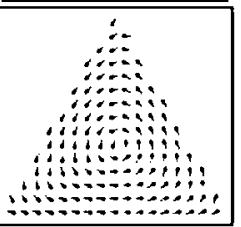

Vortex-state

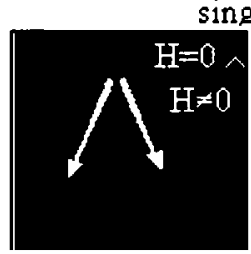

ingle domain

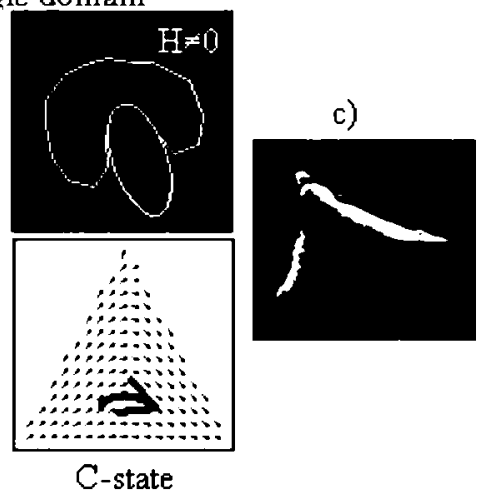

Fig. 4. (a) Classification of the contrast found in MFM imaging. (b) Magnetisation vector plots obtained from micromagnetic simulation for the vortex state, the Y-state and the C-state. For the Y-state, the grey colors correspond to the theoretical MFM contrast. (c) SEM image of $30 \mathrm{~nm}$ Ni triangles revealing the real geometry (taken at non-perpendicular incidence).

samples, MFM images were taken in an applied field as shown in Fig. 3 (bottom row). For the $10 \mathrm{~nm}$ elements, the flux closure structure seems to be stable up to rather large fields (200 Oe), before switching to a (quasi-) single domain contrast, while the $20 \mathrm{~nm}$ elements switch in a rather low field. For the $30 \mathrm{~nm}$ elements, the remanent disordered single domain contrast reorients towards the applied field upon increasing field. Here an interesting transition is observed at 25 Oe towards a (quasi-) single domain contrast, characterised by a U-shaped dark contrast along the element border. A second transition from this $\mathbf{U}$-shaped contrast into a different kind of single domain contrast is seen at 50 Oe. In total three different kinds 
of domain contrasts have been observed which are summarised in Fig. 4a (top) and which are compared to micromagnetics simulations, Fig. 4b (bottom).

\section{Magnetisation configurations}

Due to the reduced size, only a finite number of magnetic configurations are stabilised in submicron sized magnetic elements. The competition between magnetostatic and exchange energies defines two classes of configurations: a (quasi-) single domain configuration and a flux closure configuration. Which one of the two corresponds to the lowest energy configuration will depend on the element lateral size, thickness, and exact shape [6]. The (quasi-) single domain state is usually stabilised for thin elements with decreased diameter, minimising exchange energy, while the flux closure (vortex) state is stabilised for thicker and larger elements, minimising magnetostatic energy.

The single domain states have been theoretically investigated for ideal equilateral triangular Permalloy elements in Ref. [4] yielding a C-state and a Y-state, see Fig. 4c. Here, we have performed micromagnetic simulations for Ni triangles using a publicly available (ITL/NIST) 2D code: Object Oriented Micromagnetic Framework (OOMMF) [7]. This code provides a framework for solving the Landau-Lifshitz-Gilbert equation of a coupled spin system, by determining the effective field and minimising the micromagnetic energy. For the calculations we have used the material parameters corresponding to nickel (saturation magnetisation $M_{\mathrm{s}}=487 \mathrm{erg} /\left(\mathrm{Oe} \mathrm{cm}^{3}\right)$ and the exchange constant $\left.A=0.9 \times 10^{-6} \mathrm{erg} / \mathrm{cm}\right)$, as well as the dimensions for the thickness and lateral sizes corresponding to the elements investigated in the experiment. For the ideal equilateral triangular shape the vortex state as well as the C-type single domain state have been obtained, which are shown in Fig. 4b (left and right, respectively). Taking spherical borders into account such as appropriate for real triangles, see SEM image in Fig. 4c, dominantly the Y-state configuration is found, having lower energy than the vortex state. This is probably related to the narrow triangular corners, which dictate a parallel alignment of the magnetisation inside the corners. Experimentally, the MFM image of Fig. 4a left is interpreted as a flux closure state, due to the dark/bright contrast lines. The MFM image of Fig. 4a, middle, is interpreted as a Y-state, where the magnetisation flows from one triangular corner towards the two opposite corners. This creates charges of opposite sign around the corners, leading to a contrast that is similar for the calculation and the experiment. For the C-state, which can be derived from the vortex state by pushing the vortex core to one edge, no experimental counterpart has been identified. Similarly, for the experimental U-shaped contrast, no theoretical magnetisation distribution has been identified so far. More systematic studies are underway, to elucidate the role of the exact triangular element shape, derived from SEM, on (i) the ground state phase diagram describing the magnetisation configurations as a function of element thickness and lateral size 
as well as on (ii) the reversal process under in-plane fields and the corresponding remanent configuration.

\section{Conclusion}

In conclusion, a systematic study of the magnetic properties has been carried out for $\mathrm{Ni}$ triangles, prepared by nanosphere lithography. The particles are ferromagnetic and in-plane magnetised. From MFM imaging different kinds of magnetisation configurations have been identified which are attributed to a flux closure state or the Y-type quasi-single domain state. The corresponding zero field images explain the low remanence found in $M-H$ hysteresis loops. The thickness dependence of the magnetisation curves reveals an oscillating behaviour, with a remanence that first increases and then decreases. This dependence might be cor-

related to the variation of the structural properties with thickness which remains to be elucidated.

\section{Acknowledgment}

We would like to thank S. Muller for the SEM imaging and A. Popa for assisting with the micromagnetic simulations. This work was funded by the European program Nanoscale Magnetic Particles No. HPRN-CT-1999-00150.

\section{References}

[1] J.I. Martín, J. Nogués, K. Liu, J.L. Vicent, I.K. Schuller, J. Magn. Magn. Mater. 256, 449 (2003)

[2] S.Y. Chou, Proc. IEEE 85, 652 (1997).

[3] J. Rybczynski, U. Ebels, M. Giersig, Colloids Surfaces A 219, 1 (2003).

[4] D.K. Koltsov, R.P. Cowburn, M.E. Welland, J. Appl. Phys. 88, 5315 (2000).

[5] R.P. Cowburn, invited Topical Review by J. Phys. D 33, R1 (2000).

[6] L. Prejbeanu, M. Natali, L.D. Buda, U. Ebels, A. Lebib, Y. Chen, K. Ounadjela, J. Appl. Phys. 91, 7343 (2002).

[7] M.J. Donahue, D.G. Porter, OOMMF User's Guide, Version1.1bo, Interagency Report NISTIR 6376. 